[3] Guillevin L et al. Rituximab versus Azathioprine for Maintenance in ANCAAssociated Vasculitis. N Engl J Med 2014;371:1771-80.

Disclosure of Interest: None declared

DOI: 10.1136/annrheumdis-2017-eular.2302

\section{AB0596 INTERFERON-Г RELEASE ASSAY (T-SPOT.TB) IN THE DIAGNOSIS OF TUBERCULOSIS INFECTION IN PATIENTS WITH BEHCET'S DISEASE: A SINGLE CENTER EXPERIENCE IN CHINA}

W. Zheng ${ }^{1}$, X. Wu ${ }^{2}$, P. Chen ${ }^{3}$, L. Zhao ${ }^{1}$, J. Liu ${ }^{1}$, L. Zhang ${ }^{4}$, X. Liu ${ }^{4}$, Y. Zhao ${ }^{1}$, X. Zeng ${ }^{1} .{ }^{1}$ Rheumatology, Peking Union Medical College Hospital, BEIJING;

${ }^{2}$ Rheumatology, General Hospital of Tianjin Medical University, Tianjing;

${ }^{3}$ Rheumatology, Affiliated Mindong Hospital of Fujian Medical University, Fujian;

${ }^{4}$ Infectious Diseases, Peking Union Medical College Hospital, BEIJING, China

Background: In many aspects Behcet's disease (BD) is correlated with tuberculosis (TB). They may mimic each other on clinical manifestations. For example erythema nodosa, arthritis, gut lesions could be occurred in both disease and it is difficult for differential diagnosis. Some patients with newly-diagnosed BD were found to have active or latent or old TB and anti-TB therapy could relieve some of the symptoms. In etiology, it has been postulated that tubercle bacilli may act as a trigger of $\mathrm{BD}$ through the mechanism of molecular mimicking. Vice versa, defective cell-mediated immunity in BD patients may increase individual susceptibility of TB.It is extremely important to clarify TB existence or not in BD patients. Since BD patients commonly need glucocorticoid and immunosuppressant or even biologic agent such as anti-TNFa. And these medications may increase the risk of occurrence or flare of TB. But positive result of PPD shows little value in this special population due to acupuncture reaction in BD patients. In this pilot study, the values of interferon $-\gamma$ release assay (T-SPOT-TB) in diagnosing active TB in $\mathrm{BD}$ patients were explored.

Objectives: To investigate the diagnostic value of the T-SPOT.TB in BD patients complicated with tuberculosis infection.

Methods: The clinical, radiology and laboratory data were collected and analyzed in 175 hospitalized BD patients from the Peking Union Medical College Hospital between January 2010 and March 2015. Statistical analysis was carried out using IBM SPSS version 20.

Results: Of the $175 \mathrm{BD}$ patients, the positive rate of tuberculosis infection in BD patients was $34.3 \%$. The sensitivity, specificity, positive predictive value (PPV) and negative predictive value (NPV) of the T-SPOT.TB test for the diagnosis of ATB were $87.5 \%, 73 \%, 36.8 \%, 98.3 \%$, respectively. Positive likelihood ratio (PLR) and negative likelihood ratio (NLR) were 3.24 and 0.17 . The median number of SFCs in the BD-ATB group was higher than that in the BD-LTB group and BD-OTB $(p<0.001$ and $p=0.012$. By ROC method, it was suggested that 70 SFCs act as a cutoff for diagnosing BD-ATB with the sensitivity, specificity, PPV, NPV, PLR and NLR were $87.5 \%, 85 \%, 24.6 \%, 98.5 \%, 5.79$ and 0.15 , respectively. The rates of LTB infection had no significant difference between BD patients and healthy controls. Agreement between T-SPOT.TB and TST in BD patients measured by the kappa coefficient was poor ( $\mathrm{kappa}=0.37$ ). Multiple logistic regression analysis revealed that $\mathrm{BD}$ patients with positive T-SPOT.TB had the highest likelihood of ATB (OR 11.93, 95\% Cl 2.108-67.508, $p=0.005$ ).

Conclusions: T-SPOT.TB is a promising test in the diagnosis of BD complicated with tuberculosis infection, and higher number of SFCs may has a higher risk of ATB.

Disclosure of Interest: None declared

DOI: 10.1136/annrheumdis-2017-eular.4149

\section{AB0597 LOW-DOSE RITUXIMAB AS INDUCTION THERAPY FOR JAPANESE PATIENTS WITH ANCA-ASSOCIATED VASCULITIS}

Y. Takakuwa, H. Hanaoka, T. Kiyokawa, K. Ishimori, H. lida, H. Nagafuchi, T. Okazaki, S. Ozaki. Division of Rheumatology and Allergy, Department of Internal Medicine, St. Marianna University School of Medicine, Kawasaki, Japan

Background: Four times of once-weekly doses of $375 \mathrm{mg} / \mathrm{m}^{2}$ rituximab (RTX) are frequently used as remission induction therapy for ANCA-associated vasculitis (AAV). Since this regimen has been basically generated from experience of B-cell non-Hodgkins' lymphoma in the Europe and North America, appropriate dose and interval for patients with AAV in other population have been poorly investigated. Here we comprehensively analyzed the efficacy and tolerability of high or low dose regimen of RTX in Japanese patients with AAV.

Objectives: We investigated the efficacy and safety issue of low dose RTX for Japanese patients with AAV.

Methods: We retrospectively examined AAV patients who met the 2012 Chapel Hill classification from 2006 to 2016. We divided them into 2 groups, those treated with high-dose (HD) and low-dose (LD) RTX. HD RTX was the original regimen and LD RTX consisted of twice of one-weekly dose of $375 \mathrm{mg} / \mathrm{m}^{2}$. We evaluated cumulative complete remission (CR) rate and relapse-free rate for 1.5 years. CR was defined as BVAS $=0$ and relapse was defined as BVAS $\geq 1$.

Results: We evaluated 17 patients with HD and 11 patients with LD RTX. Higher percentage of elderly patients was observed in LD group $(p<0.01)$. No significant difference was found in BVAS $(p=0.49)$ and VDI $(p=0.15)$ before treatment. No significant difference was found in cumulative CR rate $(p=0.90)$ (Fig. 1A), relapse-free rate $(p=0.48)$ (Fig. 1B), B cells counts and serious adverse events. We found patients with nasal involvement and pulmonary nodule/cavity formation had higher relapse rate in LD group than those with $\operatorname{HD}$ group $(p=0.05$, and $p=0.09$ respectively).

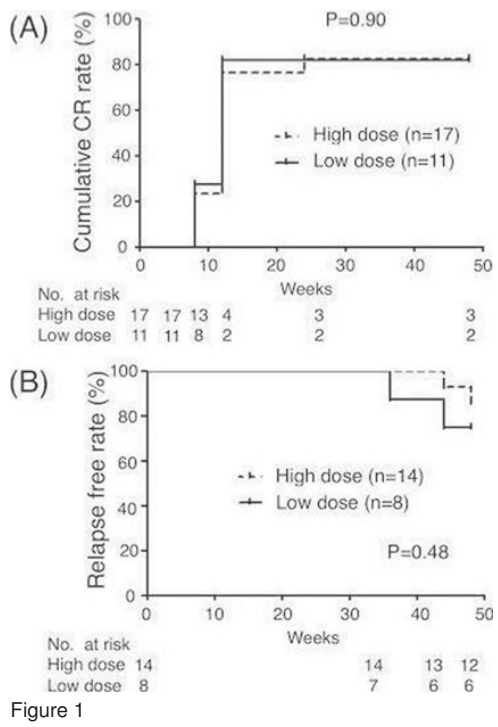

Conclusions: LD regimen of RTX, especially in elderly patients, might be effective in remission induction therapy in AAV.

References:

[1] Ozaki S, et al. Mod Rheumatol 2012; 22: 394-404.

Disclosure of Interest: None declared

DOI: 10.1136/annrheumdis-2017-eular.5410

\section{Scleroderma, myositis and related syndromes}

\section{AB0598 SERUM DEFENSIN LEVEL IN SYSTEMIC SCLEROSIS PATIENTS}

T. Emiroglu ${ }^{1}$, H. Küçük ${ }^{2}$, B. Goker ${ }^{2}$, S. Haznedaroglu ${ }^{2}$, H. Pasaoglu ${ }^{3}$,

Ö. Varan ${ }^{2}$, M.A. Öztürk ${ }^{2}$, O.T. Pasaoglu ${ }^{3}$, A. Tufan ${ }^{2} .{ }^{1}$ Department of Internal

Medicine; ${ }^{2}$ Department of Internal Medicine, Division of Rheumatology;

${ }^{3}$ Department of Biochemistry, Gazi University Faculty of Medicine, Ankara, Turkey

Background: Skleroderma is an autoimmune disease characterized by fibrosis of skin and lung as well as involvement of kidney, gastrointestinal system and heart (1). Etiology and exact mechanism of disease is poorly understood. A small number of studies have examined the role of AMPs on autoimmune diseases. It has been demonstrated that the amount of alfa- and beta-2 defensin serum levels are increased in systemic lupus erythematosus patients (2.3). Likewise, the association between AMPs and other diseases such as idiopathic pulmonary fibrosis, diffuse panbronchiolitis, pulmoner alveolar proteinosis and psoriasis has been reported (4).

Objectives: No study investigated the role of AMPs on scleroderma patients. Hence, we aimed to investigate AMP serum levels and their possible association in these patients.

Methods: There were 42 patients (40 female, mean age 42 years) and 38 healthy subjects (32 female, mean age 38 years) in the study. For SSc patients, the following data were recorded at enrollment: disease subset (limited/diffuse), autoantibodies (antinuclear, anti-centromere (ACA), and anti-scl), blood tests, erythrocyte sedimentation rate (ESR), and C-reactive protein (CRP), modified Rodnan skin score, presence and history of digital ulcers, presence and history of involvement kidney and gastrointestinal system, interstitial lung disease detected by chest HRCT and pulmonary function tests, estimated pulmonary arterial systolic pressure at echocardiography.

Results: There were 42 patients ( 40 female, mean age 42 years) and 38 healthy subjects ( 32 female, mean age 38 years) in the study. Twenty-nine of the patients had diffuse systemic sclerosis and thirteen of the patients had limited systemic sclerosis. Average disease duration is 5.5 years. Pulmonary involvement was detected in twenty patients. The levels of beta 1 and beta 2 defensin that are epitelyal defensins were higher than control group but it has not reached statistical significance. (beta-1 defensin $235 \pm 178$ vs $185 \pm 24 \mathrm{pg} / \mathrm{ml}, \mathrm{p}=0.08$ and beta2 defensin $253 \pm 453$ vs $152 \pm 101 \mathrm{pg} / \mathrm{ml}, \mathrm{p}=0,1)$. Alpha defensin levels in scleroderma patients were significantly higher than control group $(563 \pm 415$ vs $377 \pm 269 \mathrm{ng} / \mathrm{mL}, \mathrm{p}=0.02)$. In sub-group analysis patients with interstitial lung disease had a higher level of alpha defensive than those without involvement $(684 \pm 473$ vs $430 \pm 299 \mathrm{ng} / \mathrm{ml}, \mathrm{p}=0.04)$. There was an negative correlation between alfa defensin and Rodnan score $(r=0.30)$ and CRP $(r=0.34)$.

Conclusions: Alpha defensin levels in scleroderma patients were significantly higher than control group, There may be an increase in the level of alpha defensin in a cause of vasulopathy in scleroderma patients 